\title{
Thermodynamics of quantum-jump-conditioned feedback control
}

\author{
Philipp Strasberg, ${ }^{1}$ Gernot Schaller, ${ }^{1}$ Tobias Brandes, ${ }^{1}$ and Massimiliano Esposito ${ }^{2}$ \\ ${ }^{1}$ Institut für Theoretische Physik, Technische Universität Berlin, Hardenbergstrasse 36, D-10623 Berlin, Germany \\ ${ }^{2}$ Complex Systems and Statistical Mechanics, University of Luxembourg, L-1511 Luxembourg, Luxembourg
}

(Received 28 May 2013; published 3 December 2013)

\begin{abstract}
We consider open quantum systems weakly coupled to thermal reservoirs and subjected to quantum feedback operations triggered with or without delay by monitored quantum jumps. We establish a thermodynamic description of such systems and analyze how the first and second law of thermodynamics are modified by the feedback. We apply our formalism to study the efficiency of a qubit subjected to a quantum feedback control and operating as a heat pump between two reservoirs. We also demonstrate that quantum feedbacks can be used to stabilize coherences in nonequilibrium stationary states which in some cases may even become pure quantum states.
\end{abstract}

DOI: 10.1103/PhysRevE.88.062107

\section{INTRODUCTION}

Manipulating small quantum systems interacting with their environments with the help of quantum feedback control is of crucial importance for modern nanotechnologies. An important step in this direction is to understand the thermodynamics of such processes, in particular if one wants to use small nanostructures as refrigerators, heat pumps, or power sources.

The regime of weak coupling between a system and its environment is nowadays well understood [1]. The system dynamics is described by a Markovian quantum master equation (QME) which in the rotating wave approximation (RWA) gives rise to a closed rate equation for the population dynamics in the system energy eigenbasis with rates satisfying local detailed balance. The theoretical framework of stochastic thermodynamics can therefore be straightforwardly applied and provides a consistent nonequilibrium thermodynamic description for these systems [2]. Without the RWA, QMEs have a more complicated structure and different unravelings of the master equation become possible. Each leads to a different definition of the thermodynamic quantities at the trajectory level which may or may not be relevant in a given experimental setup [3-7].

In this paper, we consider open quantum systems subjected to a quantum feedback control and described by QMEs in the RWA where the notion of heat remains unambiguously defined. Classical and quantum feedback control opens a lot of exciting possibilities to control small systems. For instance, feedback can be used to transport electrons against a potential bias [8-10] or to control Brownian particles in potential traps [11-13]. From a thermodynamic point of view, feedbacks may inject energy as well as entropy into the system and may thus modify the first as well as the second law of thermodynamics. Two interesting particular cases are the mechanical work source, which injects energy but no entropy, and the Maxwell demon feedback, which injects entropy but no energy [14]. Many recent studies have analyzed and quantified the thermodynamic effect of gathering information by a measurement performed at predetermined times and operating back on the system with a time-dependent force which depends on the measurement output [15-21]. These setups should be contrasted from feedback schemes which rely on a continuous measurement of the system and which operate whenever a given signal is detected from the system.
PACS number(s): 05.70.Ln, 05.60.Gg, 05.40.-a

The thermodynamics of such feedbacks has been less studied and has only been recently considered in Refs. [10,14].

Our main objective in this study is to characterize the thermodynamic implications of a class of feedbacks which were initially introduced in quantum optics by Wiseman and Milburn [22]. A specific energy or matter transfer between a quantum system and its reservoirs is continuously monitored. Whenever the transfer event is detected a quantum operation is triggered on the system. The theoretical description of such feedback relies on the identification of jump operators in a QME which are associated with detection events, e.g., photon emission [22,23]. Such feedbacks can be used to stabilize pure quantum states (e.g., of qubits or of the photon field) as was shown theoretically [24] as well as experimentally [25-27]. Similar results were also obtained in quantum transport where jump operators correspond to electron detection events $[28,29]$.

This paper is organized as follows. In Sec. II we start by reviewing stochastic thermodynamics for open quantum systems in general and on a model of a qubit weakly coupled to two thermal reservoirs. In Sec. III we introduce the feedback scheme and analyze its effect on the first and second law. We also discuss the effect of delays in the feedback and illustrate our results on the qubit model. In Sec. IV we focus on two applications: a quantum heat pump and the stabilization of quantum mechanical coherences. Conclusions are drawn in Sec. V.

\section{STOCHASTIC THERMODYNAMICS OF OPEN QUANTUM SYSTEMS}

We consider a small system with $M$ different energy eigenstates (for instance a quantum dot, a qubit, a molecule, or a spin), which is described by the system Hamiltonian $H_{S}=\sum_{i=1}^{M} E_{i}|i\rangle\langle i|$ and which is weakly coupled to $N$ ideal thermal reservoirs at a given inverse temperature $\beta_{v}=1 / T_{v}$ ( $k_{B} \equiv 1$ throughout the paper) where $v \in\{1, \ldots, N\}$ is used as an index for the different reservoirs. We assume that the reservoirs do not interact directly and solely focus on energy transfer in this paper. For simplicity we exclude matter transfer which would require to include the chemical potentials of the reservoirs $\mu_{v}$ in the description. We also assume that every transition between the energy eigenstates of the system is 
triggered by the absorption/emission of an energy quantum from/to a particular reservoir $v$.

\section{A. Dynamics}

In the weak coupling regime where the Born-Markov approximation is justified, the time evolution of the system density matrix $\rho$ follows the Markovian QME [1]:

$$
\frac{\partial}{\partial t} \rho(t)=\mathcal{W} \rho(t)
$$

The generator $\mathcal{W}$ is a superoperator (operator) in Hilbert (Liouville) space. To fix the notation we use latin letters $i, j, k$ to label the energy levels of the system and $|i\rangle,|j\rangle,|k\rangle$ are the energy eigenstates in the system Hilbert space. In Liouville space we order the elements of the density matrix into the vector $\left(\rho_{\text {pop }}, \rho_{\text {coh }}\right)$ where $\rho_{\text {pop }}=\left(p_{1}, \ldots, p_{M}\right)$ describes the populations $p_{i}=\langle i|\rho| i\rangle$ and $\rho_{\text {coh }}=\left(\rho_{12}, \rho_{21}, \ldots, \rho_{M-1 M}, \rho_{M M-1}\right)$ is the vector of the $M(M-1)$ coherences $\rho_{i j}=\langle i|\rho| j\rangle, i \neq j$. Consequently, the trace of a matrix becomes a sum over the first $M$ entries of the corresponding vector in Liouville space. Furthermore, we order the indexes $\{i\}$ such that $i>j$ implies also $E_{i}>E_{j}$. Thus, we exclude the possibility of degeneracies in the system. To use an intuitive notation we will also use the indexes $i, j, k$ to refer to the populations in Liouville space, i.e., whenever there is a sum over $i, j, k$ it runs from 1 to $M$. The corresponding states in Liouville space are denoted by $|i\rangle\rangle,|j\rangle\rangle,|k\rangle\rangle$. We will not introduce a notation for the coherences because we do not need them.

In the energy eigenbasis of the system Hamiltonian, due to the RWA the generator has a block structure of the form

$$
\mathcal{W}=\left(\begin{array}{cc}
\mathcal{W}_{\text {pop }} & 0 \\
0 & \mathcal{W}_{\text {coh }}
\end{array}\right)
$$

which shows that population and coherences evolve independently from each other in that basis. In fact the populations obey a rate equation $\partial_{t} \rho_{\text {pop }}=\mathcal{W}_{\text {pop }} \rho_{\text {pop }}$, while the coherences are exponentially damped and vanish at steady state. The generator of the populations can be made more explicit,

$$
\mathcal{W}_{\text {pop }}=\mathcal{W}_{0}+\sum_{v} \sum_{i>j}\left(\mathcal{J}_{j \rightarrow i}^{v}+\overline{\mathcal{J}}_{i \rightarrow j}^{v}\right),
$$

where $\mathcal{J}_{j \rightarrow i}^{v}\left(\overline{\mathcal{J}}_{i \rightarrow j}^{v}\right)$ is a jump-superoperator responsible for a quantum jump upwards from level $j$ to $i$ (downwards from level $i$ to $j$ ) corresponding to an energy $E_{i}-E_{j}>0$ entering (exiting) the system from (to) reservoir $v$ as illustrated in Fig. 1. Mathematically, these jump operators can be expressed as

$$
\left.\mathcal{J}_{j \rightarrow i}^{v}=\gamma_{j \rightarrow i}^{v}|i\rangle\right\rangle\left\langle\left\langle j\left|, \quad \overline{\mathcal{J}}_{i \rightarrow j}^{v}=\bar{\gamma}_{i \rightarrow j}^{v}\right| j\right\rangle\right\rangle\langle\langle i|,
$$

where $\gamma_{j \rightarrow i}^{v}$ and $\bar{\gamma}_{i \rightarrow j}^{v}$ are rates satisfying local detailed balance:

$$
\ln \left(\gamma_{j \rightarrow i}^{\nu} / \bar{\gamma}_{i \rightarrow j}^{v}\right)=-\beta_{\nu}\left(E_{i}-E_{j}\right) .
$$

If we have correctly identified all the jumps the remaining part of the generator $\mathcal{W}_{0}$ has the form

$$
\mathcal{W}_{0}=-\sum_{\nu} \sum_{i>j}\left(\gamma_{j \rightarrow i}^{v}|j\rangle\right\rangle\left\langle\left\langle j\left|+\bar{\gamma}_{i \rightarrow j}^{v}\right| i\right\rangle\right\rangle\langle\langle i|) .
$$

The probability current associated to the transition $(\nu,(i, j))$ is given by (see Appendix A)

$$
I_{(i, j)}^{v}(t)=\gamma_{j \rightarrow i}^{v} p_{j}(t)-\bar{\gamma}_{i \rightarrow j}^{v} p_{i}(t) .
$$

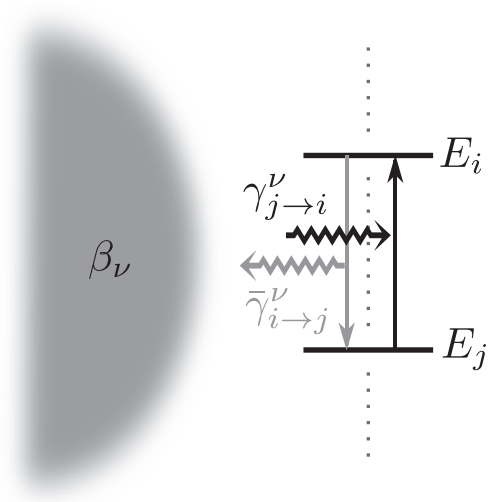

FIG. 1. Illustration of a possible transition between two energy levels $E_{i}$ and $E_{j}$ of the system by absorption of an energy quantum (in black) or an emission of an energy quantum (in gray) from or into the thermal reservoir $v$.

Often however, we are only interested in the long time steady state behavior of the system where the probabilities $p_{j}(t)$ become time independent and fulfill $\sum_{j}\left(\mathcal{W}_{\text {pop }}\right)_{i j} p_{j}=0$ for all $i$. In this case, we adopt the simple notation $p_{j}=$ $\lim _{t \rightarrow \infty} p_{j}(t), I_{(i, j)}^{\nu}=\lim _{t \rightarrow \infty} I_{(i, j)}^{\nu}(t)$, etc., i.e., we drop the time dependence if we talk about the steady state.

\section{B. Thermodynamics}

Since we have identified the currents for each transition, we can now introduce the heat flow from reservoir $v$ as

$$
\dot{Q}^{(v)}(t)=\sum_{i>j}\left(E_{i}-E_{j}\right) I_{(i, j)}^{v}(t) .
$$

It is by definition positive if it enters the system. We denote the change in the system energy by

$$
\dot{E}(t) \equiv \frac{d}{d t} \operatorname{tr}\left[\mathcal{H}_{S} \rho\right]=\sum_{i} E_{i} \dot{p}_{i}(t),
$$

where $\mathcal{H}_{S}$ is a formal expression of the Hamiltonian in Liouville space with entries $\left.\mathcal{H}_{S}=\sum_{i} E_{i}|i\rangle\right\rangle\langle\langle i|$. The first law of thermodynamics now demands that the change in system energy is balanced by the heat flows into the system (see Appendix B),

$$
\dot{E}(t)=\sum_{v} \dot{Q}^{(v)}(t) .
$$

At steady state, the left hand side vanishes and the first law becomes $\sum_{v} \dot{Q}^{(v)}=0$.

We will use the Shannon entropy of the systems populations to characterize the entropy of the system. We note that due to the structure of Eq. (2), at steady state the von Neumann entropy of the system coincides with the Shannon entropy of the system. As customary in stochastic thermodynamics, the change in Shannon entropy of the system can be split in two parts [2]:

$$
\frac{d}{d t} S(t)=-\frac{d}{d t} \sum_{i} p_{i}(t) \ln p_{i}(t)=\dot{S}_{\mathbf{i}}(t)+\dot{S}_{\mathbf{e}}(t),
$$

where $\dot{S}_{\mathbf{i}}(t)$ is the non-negative entropy production which quantifies the irreversibility of the dynamics and $\dot{S}_{\mathbf{e}}(t)$ is 
the entropy flow arising from heat exchanges with the environments. More explicitly

$$
\begin{gathered}
\dot{S}_{\mathbf{i}}(t)=\sum_{v} \sum_{i, j} \mathcal{W}_{i j}^{(v)} p_{j}(t) \ln \frac{\mathcal{W}_{i j}^{(v)} p_{j}(t)}{\mathcal{W}_{j i}^{(v)} p_{i}(t)} \geqslant 0, \\
\dot{S}_{\mathbf{e}}(t)=\sum_{\nu} \sum_{i, j} \mathcal{W}_{i j}^{(v)} p_{j}(t) \ln \frac{\mathcal{W}_{j i}^{(v)}}{\mathcal{W}_{i j}^{(v)}},
\end{gathered}
$$

where $\mathcal{W}_{i j}^{(v)}$ denotes the matrix elements of the population generator (3) associated to transitions triggered by the reservoir $\nu$. In the case without feedback they are simply determined by the rates in Eq. (4).

Using the local detailed balance relation in Eq. (5), it is easy to show that the entropy flow can be expressed as (minus) the reversible entropy changes in the reservoirs $\dot{S}_{\mathbf{e}}(t)=\sum_{v} \dot{Q}^{(v)}(t) / T_{v}$. Hence,

$$
\dot{S}_{\mathbf{i}}(t)=\dot{S}(t)-\sum_{v} \frac{\dot{Q}^{(v)}(t)}{T_{v}} \geqslant 0,
$$

which corresponds to the second law of thermodynamics. At steady state the change in system entropy vanishes, $\dot{S}=0$, such that $\dot{S}_{\mathbf{i}}=-\dot{S}_{\mathbf{e}}$.

\section{Qubit model}

As our paradigmatic model we consider a qubit weakly coupled to two bosonic reservoirs $L$ and $R$. Such spin-boson models have been studied extensively in the literature for instance to understand heat pumps and thermal transport through molecules [30-32]. We consider phonon reservoirs, but photons could be considered as well. The total Hamiltonian is the sum of three contributions,

$$
\begin{gathered}
H=H_{S}+H_{B}+V, \\
H_{S}=\frac{\Omega}{2}(|1\rangle\langle 1|-| 0\rangle\langle 0|), \\
H_{B}=\sum_{\nu \in\{L, R\}} \sum_{\mathbf{q}} \omega_{\mathbf{q} \nu} b_{\mathbf{q} v}^{\dagger} b_{\mathbf{q} v}, \\
V=\sum_{\nu \in\{L, R\}} \sum_{\mathbf{q}} T_{\mathbf{q} v}\left(b_{\mathbf{q} v}^{\dagger}|0\rangle\left\langle 1\left|+b_{\mathbf{q} v}\right| 1\right\rangle\langle 0|\right),
\end{gathered}
$$

where $\omega_{\mathbf{q} v}>0, \Omega>0, T_{\mathbf{q} v} \in \mathbb{R}$, and $b_{\mathbf{q} v}$ are bosonic annihilation operators. We considered the interaction in the RWA.

The master equation of this system in the Born-Markov approximation is well known $[1,22]$. In the basis $\left(p_{0}=\right.$ $\left.\langle 0|\rho| 0\rangle, p_{1}=\langle 1|\rho| 1\rangle, \rho_{01}, \rho_{10}\right)$ the generator has the structure (2) where

$$
\begin{gathered}
\mathcal{W}_{\text {pop }}=\sum_{\nu}\left(\begin{array}{cc}
-\gamma_{\nu} & \bar{\gamma}_{\nu} \\
\gamma_{\nu} & -\bar{\gamma}_{\nu}
\end{array}\right), \\
\mathcal{W}_{\text {coh }}=\left(\begin{array}{cc}
i \Omega^{\prime}-\sum_{\nu} \frac{\gamma_{v}+\bar{\gamma}_{v}}{2} & 0 \\
0 & -i \Omega^{\prime}-\sum_{\nu} \frac{\gamma_{v}+\bar{\gamma}_{v}}{2}
\end{array}\right) .
\end{gathered}
$$

The rates $\gamma_{\nu}, \bar{\gamma}_{\nu}$ are determined by the Bose distribution $n_{v}(\Omega)=\left(e^{\beta_{\nu} \Omega}-1\right)^{-1}$ evaluated at the level splitting: $\gamma_{\nu}=$ $\Gamma_{v} n_{v}(\Omega), \bar{\gamma}_{v}=\Gamma_{v}\left[1+n_{v}(\Omega)\right]$ with $\Gamma_{v}>0 . \Omega^{\prime}$ is the renormalized level splitting due to the Lamb shifts.

We now focus on the steady state behavior of the system. We find that $p_{0}=1-p_{1}=\left(\sum_{v} \bar{\gamma}_{v}\right) /\left[\sum_{v}\left(\gamma_{v}+\bar{\gamma}_{v}\right)\right]$ and the coherences vanish $\rho_{01}=\rho_{10}=0$. The probability current (7) is given by $I^{\nu}=\gamma_{\nu} p_{0}-\bar{\gamma}_{\nu} p_{1}$ and the corresponding heat flow becomes $\dot{Q}^{(v)}=\Omega I^{\nu}$. Consequently, the first and second law of thermodynamics read

$$
\text { I. } \dot{Q}^{L}+\dot{Q}^{R}=0, \quad \text { II. }\left(\beta_{R}-\beta_{L}\right) \dot{Q}^{L} \geqslant 0 \text {. }
$$

The second law expresses the fact that on average the phonons are flowing from the hot to the cold reservoir.

\section{THERMODYNAMICS OF WISEMAN-MILBURN FEEDBACKS}

A feedback describes the situation in which a system is measured and according to the measurement output a certain operation is performed on it. In our case the identification of jump processes defines a weak measurement of the system by the reservoirs. Note that also "no signal," i.e., the time between two subsequent jumps, reveals information about the system. The idea of Wiseman and Milburn was now to use the signal of detection events to trigger control operations on the system $[22,23]$.

\section{A. Control operations}

To describe the situation with feedback we introduce control superoperators $\mathcal{C}_{j \rightarrow i}^{\nu}\left(\overline{\mathcal{C}}_{i \rightarrow j}^{\nu}\right)$, which act on the system after a certain absorption (emission) process in the system has been induced by the reservoir $v$. Most of the time we will assume that the control operation acts instantaneously after a jump on the system, i.e., the feedback is much faster than all other relevant time scales of the system (the case of a finite delay will be treated in Sec. III C). The resulting effective generator can be written as $[22,23]$

$$
\mathcal{W}^{C}=\mathcal{W}_{0}+\sum_{\nu} \sum_{i>j}\left(\mathcal{C}_{j \rightarrow i}^{v} \mathcal{J}_{j \rightarrow i}^{v}+\overline{\mathcal{C}}_{i \rightarrow j}^{v} \overline{\mathcal{J}}_{i \rightarrow j}^{v}\right) .
$$

Using the form of the jump operators, Eq. (4), we see that this generator has a block structure of the form

$$
\mathcal{W}^{C}=\left(\begin{array}{cc}
\mathcal{W}_{\mathrm{pop}}^{C} & 0 \\
\mathcal{W}_{c p}^{C} & \mathcal{W}_{\mathrm{coh}}
\end{array}\right)
$$

The populations still evolve independently from the coherences, but the coherences get affected by the populations. This implies that such a feedback is able to build up coherences in the steady state, as we will see in detail in Sec. IV B.

The quantum control operation is chosen as a unitary operation $U_{\mathcal{C}}\left(U_{\mathcal{C}}^{\dagger} U_{\mathcal{C}}=1\right)$ in the Hilbert space of the system: $\mathcal{C} \rho \leftrightarrow U_{\mathcal{C}} \varrho U_{\mathcal{C}}^{\dagger}$, where $\varrho$ denotes the density matrix acting in Hilbert space whereas $\rho$ denotes the corresponding vector in Liouville space. Defining the transition probability due to the control operation $\mathcal{C}_{k i} \equiv\langle\langle k|\mathcal{C}| i\rangle\rangle=\left|\left\langle k\left|U_{\mathcal{C}}\right| i\right\rangle\right|^{2}$ from population $i$ to population $k$, we find that

$$
\sum_{k=1}^{M} \mathcal{C}_{k i}=\sum_{i=1}^{M} \mathcal{C}_{k i}=1
$$



as

The effective population generator can be explicitly written

$$
\begin{aligned}
\mathcal{W}_{\text {pop }}^{C}= & \sum_{\nu} \sum_{i>j} \gamma_{j \rightarrow i}^{v}\left(\sum_{k}\left(\mathcal{C}_{j \rightarrow i}^{v}\right)_{k i}|k\rangle\right\rangle\langle\langle j|-| j\rangle\rangle\langle\langle j|) \\
& +\sum_{\nu} \sum_{i>j} \bar{\gamma}_{i \rightarrow j}^{v}\left(\sum_{k}\left(\overline{\mathcal{C}}_{i \rightarrow j}^{v}\right)_{k j}|k\rangle\right\rangle\langle\langle i|-| i\rangle\rangle\langle\langle i|) .
\end{aligned}
$$

The dynamics of the populations can be interpreted as follows. Immediately after the detection of a jump from $|j\rangle\rangle \rightarrow|i\rangle\rangle$, the control operation generates a further "jump" from $|i\rangle\rangle \rightarrow$ $|k\rangle\rangle$ with probability $\left(\mathcal{C}_{j \rightarrow i}^{v}\right)_{k i}$. The matrix elements of (25) do not satisfy local detailed balance anymore. In absence of feedback $\left(\mathcal{C}_{j \rightarrow i}^{v}\right)_{k i}=\delta_{k i}$ and we recover the generator (3). If the feedback operation commutes with the system Hamiltonian, $\left[C, H_{S}\right]=0$, the generator remains unaffected by the control operation: $\mathcal{W}^{C}=\mathcal{W}$.

\section{B. Thermodynamics of feedback}

The probability current associated with the $v$ th reservoir induced transition $|j\rangle\rangle \rightarrow|i\rangle\rangle$ is given by

$$
I_{(i, j)}^{v}(t)=\gamma_{j \rightarrow i}^{v} p_{j}(t)-\bar{\gamma}_{i \rightarrow j}^{v} p_{i}(t) .
$$

This result is derived using counting statistics methods in Appendix A. Not surprisingly, we get the same expression as without feedback, Eq. (7), but where the steady state probabilities are obtained from the generator $\mathcal{W}^{C}$ instead of $\mathcal{W}$. The heat flow is consequently given by

$$
\dot{Q}^{(v)}(t)=\sum_{i>j}\left(E_{i}-E_{j}\right) I_{(i, j)}^{\nu}(t)
$$

as in Eq. (8).

The rate of energy injection by the control operation after a transition $|j\rangle\rangle \rightarrow|i\rangle\rangle$ triggered by reservoir $v$ can be obtained from the difference between the energy of the system after the transition and the subsequent control operation and the energy of the system right after the transition but before the control operation:

$$
\begin{aligned}
\left(\dot{\mathcal{F}}_{E}^{(v)}\right)_{(i, j)}(t)= & \operatorname{tr}\left[\mathcal{H}_{S}\left(\mathcal{C}_{j \rightarrow i}^{v}-1\right) \mathcal{J}_{j \rightarrow i}^{v} \rho(t)\right] \\
& +\operatorname{tr}\left[\mathcal{H}_{S}\left(\overline{\mathcal{C}}_{i \rightarrow j}^{v}-1\right) \overline{\mathcal{J}}_{i \rightarrow j}^{v} \rho(t)\right] .
\end{aligned}
$$

It is positive if the feedback in average injects energy into the system and it is zero in absence of feedback (i.e., when the $\mathcal{C}_{i \rightarrow j}^{v}$ are the identity operator).

The change in the system energy has now to be balanced by the heat flows (27) and the energy injected by the feedback (28). The first law of thermodynamics thus gets modified according to

$$
\dot{E}(t)=\sum_{v}\left(\dot{Q}^{(v)}(t)+\dot{\mathcal{F}}_{E}^{(v)}(t)\right),
$$

where $\dot{\mathcal{F}}_{E}^{(v)}(t) \equiv \sum_{i>j}\left(\dot{\mathcal{F}}_{E}^{(v)}\right)_{(i, j)}(t)$. This result is explicitly derived in Appendix B.

We now turn to the second law. Since the population generator is still a proper rate matrix, the change in time of the Shannon entropy can be again split as in Eq. (11) and the entropy production $\dot{S}_{\mathbf{i}}$ and the entropy flow $\dot{S}_{\mathbf{e}}$ are defined as usual as [2]

$$
\begin{gathered}
\dot{S}_{\mathbf{i}}(t)=\sum_{\nu} \sum_{i, j}\left(\mathcal{W}^{C}\right)_{i j}^{(v)} p_{j}(t) \ln \frac{\left(\mathcal{W}^{C}\right)_{i j}^{(v)} p_{j}(t)}{\left(\mathcal{W}^{C}\right)_{j i}^{(v)} p_{i}(t)} \geqslant 0, \\
\dot{S}_{\mathbf{e}}(t)=\sum_{\nu} \sum_{i, j}\left(\mathcal{W}^{C}\right)_{i j}^{(v)} p_{j}(t) \ln \frac{\left(\mathcal{W}^{C}\right)_{j i}^{(v)}}{\left(\mathcal{W}^{C}\right)_{i j}^{(v)}}
\end{gathered}
$$

We remark however that, since coherences survive at steady state, the von Neumann entropy $\mathcal{S}=-\operatorname{tr}[\rho \ln \rho]$ is not equivalent anymore to the Shannon entropy. This suggests that (30) is not the only possible choice of entropy production. An approach based on the splitting of the evolution of the system von Neumann entropy as an entropy production and entropy flow term (in the basis diagonalizing the system density matrix) as proposed in [3] could also have been attempted. In any case, the choice of entropy production (30) constitutes a non-negative quantity which only cancels when all probability currents between pairs of populations due to a transition from a reservoir $v$ and its corresponding feedback operation vanish: $\left(\mathcal{W}^{C}\right)_{i j}^{(\nu)} p_{j}=\left(\mathcal{W}^{C}\right)_{j i}^{(\nu)} p_{i}$

This means that the modified entropy flow reads now

$$
\dot{S}_{\mathbf{e}}(t)=\sum_{v} \frac{\dot{Q}^{(v)}(t)}{T_{v}}-\dot{\mathcal{F}}_{S}(t),
$$

where $\dot{\mathcal{F}}_{S}(t)$ characterizes the influence of the feedback on the entropy balance (or the "second law of thermodynamics"). Its explicit expression is given in Appendix B. This information flow $\dot{\mathcal{F}}_{S}(t)$ will be useful to define notions of feedback efficiency as we will see in Sec. IV A. In the steady state regime we have once again that

$$
\dot{S}_{\mathbf{i}}=-\dot{S}_{\mathbf{e}}=-\sum_{v} \frac{\dot{Q}^{(v)}}{T_{v}}+\dot{\mathcal{F}}_{S} \geqslant 0 .
$$

In summary, we have introduced two new quantities $\dot{\mathcal{F}}_{E}$ and $\dot{\mathcal{F}}_{S}$ to take into account the influence of the feedback on the energy balance (the first law) and on the entropy balance (the second law) on the system. Both are additive in terms of the reservoirs: $\dot{\mathcal{F}}_{E, S}=\sum_{\nu} \dot{\mathcal{F}}_{E, S}^{(v)}$. We can distinguish two limiting regimes of feedback control, an energy dominated regime for $\left|\dot{\mathcal{F}}_{E}\right| \gg 0$ and $\dot{\mathcal{F}}_{S} \approx 0$ and an entropy (or information) dominated feedback for $\dot{\mathcal{F}}_{E} \approx 0$ and $\left|\dot{\mathcal{F}}_{S}\right| \gg 0$. The latter corresponds to the class of "Maxwell demon feedback" introduced in Ref. [14]. The Wiseman-Milburn feedbacks presented here can however never fully operate as "Maxwell demon feedback" since by construction the control operation (if it does not commute with the system Hamiltonian) always injects or removes energy from the system. This statement is expected not to hold any longer if we consider quantum systems with degenerate states because the feedback operation may give rise to nontrivial effects without energy consumption in the degenerate subspace. The present thermodynamic analysis is summarized in Fig. 2.

\section{Delayed feedback control}

In this section we briefly discuss how situations with a finite delay between the measurement and the control operation can 


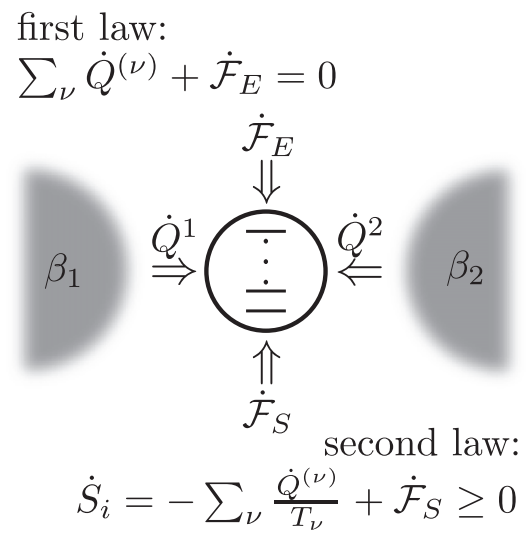

FIG. 2. Summary of the modified first and second law for a system at steady state subjected to feedback control in contact with two reservoirs. Both the first and second law are modified due to the rate of energy injection $\dot{\mathcal{F}}_{E}$ by the feedback and the information flow $\dot{\mathcal{F}}_{S}$.

be treated within our framework. A detailed discussion of the thermodynamic influence for time-delayed feedback control is however beyond the scope of the present paper.

The theory was developed in Ref. [33] where it was shown that an arbitrary delay time still leads to a nonMarkovian master equation description provided one performs the so-called "control-skipping assumption." This assumption demands that the control operation is skipped when another jump is monitored during the delay. The resulting master equation reads

$$
\begin{aligned}
\dot{\rho}(t)= & \left(\mathcal{W}_{0}+\sum_{\alpha} \mathcal{J}_{\alpha}\right) \rho(t) \\
& +\sum_{\alpha}\left(\mathcal{C}_{\alpha}-1\right) e^{\mathcal{W}_{0} \tau_{\alpha}} \mathcal{J}_{\alpha} \theta\left(t-\tau_{\alpha}\right) \rho\left(t-\tau_{\alpha}\right) .
\end{aligned}
$$

Here, the set $\{\alpha\}$ enumerates all the possible jumps $\mathcal{J}_{\alpha}$ which are followed by some control operation $\mathcal{C}_{\alpha}$ after a certain delay time $\tau_{\alpha}$ and $\theta\left(t-\tau_{\alpha}\right)$ denotes the Heaviside step function.

We verify that we recover the master equation (1) with (3) in absence of feedback (when $\mathcal{C}_{\alpha}=1$ for all $\alpha$ ) and the master equation (1) with (22) in case of vanishing delay time $\tau_{\alpha}=0$. For an infinite delay time, using (6) we have that $\lim _{\tau_{\alpha} \rightarrow \infty} e^{\mathcal{W}_{0} \tau_{\alpha}}=0$ and thus we recover our master equation without feedback. This makes sense since for an infinite delay time the control operation is never performed due to the "control-skipping assumption."

We would like to define heat, work, and entropy for the nonMarkovian equation (34). However, the theory of stochastic thermodynamics for non-Markovian dynamics requires some care [34-36] and is not as straightforward as its Markovian counterpart. We will focus therefore exclusively on the steady state behavior.

Let us consider Eq. (34) in Laplace space [the Laplace transform of an arbitrary function of time $f(t)$ is defined as $\left.\hat{f}(z) \equiv \int_{0}^{\infty} d t e^{-z t} f(t)\right]$. We get [33]

$$
z \hat{\rho}(z)-\rho(0)=\mathcal{W}_{\text {delay }}(z) \hat{\rho}(z),
$$

where

$$
\mathcal{W}_{\text {delay }}(z)=\mathcal{W}_{0}+\sum_{\alpha}\left[1+\left(\mathcal{C}_{\alpha}-1\right) e^{\left(\mathcal{W}_{0}-z\right) \tau_{\alpha}}\right] \mathcal{J}_{\alpha}
$$

This system exhibits one (or several) nontrivial steady states $\rho \equiv \lim _{z \searrow 0} z \hat{\rho}(z)=\lim _{t \rightarrow \infty} \rho(t)$ if $\mathcal{W}_{\text {delay }}(0)$ has one (or several) zero eigenvalues. For large $t$ when the system is close to steady state we have $\rho\left(t-\tau_{\alpha}\right) \approx \rho(t)$ and $\theta\left(t-\tau_{\alpha}\right)=1$ for all $\tau_{\alpha}$ and thus

$$
0 \approx \frac{\partial}{\partial t} \rho(t)=\mathcal{W}_{\text {delay }}(0) \rho(t),
$$

where $\mathcal{W}_{\text {delay }}(0)$ is a well-defined Markovian generator. This generator can be interpreted as a feedback generator (22) without delay if we choose as a control operation $\tilde{\mathcal{C}}_{\alpha}=1+$ $\left(\mathcal{C}_{\alpha}-1\right) e^{\mathcal{W}_{0} \tau_{\alpha}}$. This operation still fulfills the condition (24). Furthermore, since we still have an additive structure of the form $\mathcal{W}_{\text {delay }}=\sum_{\nu} \mathcal{W}_{\text {delay }}^{(v)}$, we can define the heat flow, the energy injected by the feedback, and the entropy production, in the same way as before for Markovian dynamics. We will use Eq. (37) to investigate numerically the impact of a time delay on the thermodynamics in Sec. IV.

\section{Qubit model}

We reconsider the qubit weakly coupled to two thermal reservoirs presented in Sec. II C. Four different types of jumps can occur in this system: the qubit can absorb $(+)$ or emit $(-)$ a phonon from or into the $v$ th reservoir. Upon detection of these jumps the qubit is subjected to a quantum control operation $\mathcal{C}_{ \pm}^{v}$ performing a unitary operation $\mathcal{C}_{ \pm}^{v} \rho \leftrightarrow U_{ \pm}^{v} \varrho\left(U_{ \pm}^{v}\right)^{\dagger}$, where $U_{ \pm}^{v} \equiv \exp \left[-i \alpha_{ \pm}^{v}(|0\rangle\langle 1|+| 1\rangle\langle 0|)\right]$ rotates the qubit around the $x$ axis on the Bloch sphere by an angle $2 \alpha_{ \pm}^{v}$. Therefore, instead of being in the ground or excited state right after the emission or absorption of a phonon, the system ends up in a superposition of energy eigenstates due to the control operation. The generator with feedback has the structure (23) with

$$
\begin{aligned}
\mathcal{W}_{\mathrm{pop}}^{C} & =\sum_{\nu}\left(\begin{array}{cc}
-\gamma_{\nu} \cos ^{2} \alpha_{+}^{\nu} & \bar{\gamma}_{\nu} \cos ^{2} \alpha_{-}^{\nu} \\
\gamma_{\nu} \cos ^{2} \alpha_{+}^{\nu} & -\bar{\gamma}_{\nu} \cos ^{2} \alpha_{-}^{\nu}
\end{array}\right), \\
\mathcal{W}_{c p}^{C} & =\frac{i}{2} \sum_{\nu}\left(\begin{array}{cc}
-\gamma_{\nu} \sin 2 \alpha_{+}^{\nu} & \bar{\gamma}_{\nu} \sin 2 \alpha_{-}^{\nu} \\
\gamma_{\nu} \sin 2 \alpha_{+}^{\nu} & -\bar{\gamma}_{\nu} \sin 2 \alpha_{-}^{v}
\end{array}\right),
\end{aligned}
$$

whereas $\mathcal{W}_{\text {coh }}^{C}$ remains unaffected by the feedback and is thus given by (20).

According to Eq. (29), the first law in presence of feedback reads

$$
\dot{E}(t)=\dot{Q}^{L}(t)+\dot{\mathcal{F}}_{E}^{L}(t)+\dot{Q}^{R}(t)+\dot{\mathcal{F}}_{E}^{R}(t),
$$

where the rate of energy injection due to feedback (28) is given by

$$
\dot{\mathcal{F}}_{E}^{(v)}(t)=\Omega\left[I_{F}^{v}(t)-I^{v}(t)\right] .
$$

Here, we introduced the effective current $I_{F}^{\nu}(t)=$ $\cos ^{2} \alpha_{+}^{\nu} \gamma_{\nu} p_{0}(t)-\cos ^{2} \alpha_{-}^{\nu} \bar{\gamma}_{\nu} p_{1}(t)$. This current corresponds to a fictitious heat current one would blindly associate to the rate matrix (38) if one did not know about the feedback. At steady state $\dot{E}=0$ and the first law can be rewritten as $I_{F}^{L}+I_{F}^{R}=0$. The steady state of the qubit is given for completeness in Appendix C.

At steady state, the second law of thermodynamics in presence of feedback is given by (33), where the entropy 
current generated by the feedback reads

$$
\dot{\mathcal{F}}_{S}=\sum_{\nu} \frac{\dot{Q}^{(\nu)}}{T_{\nu}}+\left(-\frac{\Omega}{T_{L}}+\frac{\Omega}{T_{R}}+f_{L}-f_{R}\right) I_{F}^{L} .
$$

We introduced $f_{v}=\ln \frac{\cos ^{2} \alpha_{+}^{v}}{\cos ^{2} \alpha^{v}}$. More explicitly, this means that the entropy production is given by

$$
\dot{S}_{\mathbf{i}}=\left(-\frac{\Omega}{T_{L}}+\frac{\Omega}{T_{R}}+f_{L}-f_{R}\right) I_{F}^{L} \geqslant 0 .
$$

The stationary regime in presence of a finite time delay can also be considered. Using $\mathcal{W}_{\text {delay }}(0)$ from Eqs. (36) and (37) we can calculate the steady state probabilities $p_{0}\left(\tau_{\alpha}\right), p_{1}\left(\tau_{\alpha}\right)$ for arbitrary delay times $\tau_{\alpha}$. They are unique in this model. Using the full counting statistics methods from Appendix A, we can evaluate the heat flows $\dot{Q}^{(v)}$ from which we can directly infer that $\dot{\mathcal{F}}_{E}=-\dot{Q}^{L}-\dot{Q}^{R}$ due to the first law. Furthermore, using Eq. (30) to calculate the entropy production, we also easily find $\dot{\mathcal{F}}_{S}=\dot{S}_{\mathbf{i}}+\dot{Q}^{L} / T_{L}+\dot{Q}^{R} / T_{R}$.

\section{APPLICATIONS}

In this section we will focus on two particular applications of the feedback scheme developed above. We will first study a quantum controlled heat pump and use our formalism to define its efficiency. We will also numerically analyze the effect of finite delay times in the feedback. Then we will study how efficient the feedback can stabilize pure quantum states in the qubit.

\section{A. Heat pump}

A heat pump is a device operating between two thermal reservoirs and using work to deliver heat to the hot reservoir. For definiteness we choose $T_{L}>T_{R}$. Other thermodynamic engines such as a refrigerator or a power source can be treated in a very similar way.

The efficiency of a conventional heat pump is characterized by the coefficient of performance which quantifies how much heat can be transferred to the hot reservoir $-\dot{Q}^{L} \geqslant 0$ by using a particular amount of work $\dot{W}>0$ :

$$
\kappa \equiv \frac{-\dot{Q}^{L}}{\dot{W}} \leqslant \frac{T_{L}}{T_{L}-T_{R}} \equiv \frac{1}{\eta_{C}},
$$

where $\eta_{C}$ is the Carnot efficiency. It is bounded between zero and the inverse Carnot efficiency.

In our setup, one would be tempted to replace the conventional work source by the external source of energy $\dot{\mathcal{F}}_{E}$ injected by the feedback, and thus to define

$$
\tilde{\kappa} \equiv \frac{-\dot{Q}_{L}}{\dot{\mathcal{F}}_{E}} .
$$

While meaningful this quantity is not bounded by the theory and as we will see can become greater than $1 / \eta_{C}$. Nevertheless, our formalism can help us define a meaningful bounded coefficient characterizing the efficiency of our heat pump. Indeed, the feedback not only injects energy into the system but also an information flow $\dot{\mathcal{F}}_{S}$. Using the first law (29) at steady state we can rewrite the second law (33) as

$$
T_{R} \dot{S}_{\mathbf{i}}=\dot{Q}^{L} \eta_{C}+\dot{\mathcal{F}}_{E}+T_{R} \dot{\mathcal{F}}_{S}
$$
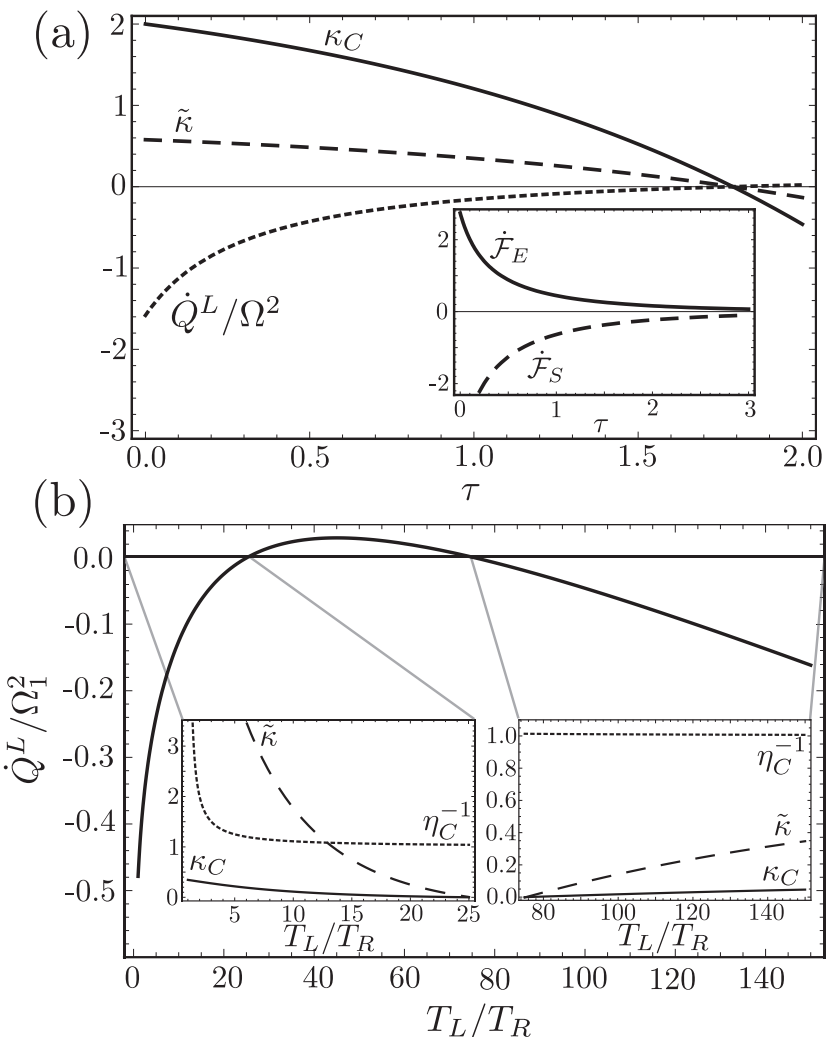

FIG. 3. (a) Qubit model: $\kappa_{C}$, Eq. (46), (solid line) and $\kappa$, Eq. (44) (dashed line), as a function of the delay time $\tau$ for $T_{L}=1$ and $T_{R}=$ $1 / 2$ (thus, $\eta_{C}^{-1}=2$ ). The dotted line represents $\dot{Q}_{L}$. As the feedback parameters we choose $\alpha_{+}^{v}=0$ and $\alpha_{-}^{v}=\pi / 2$. Inset: Energy and entropy injected by the feedback $\dot{\mathcal{F}}_{E}$ (solid line) and $\dot{\mathcal{F}}_{S}$ (dashed line) as a function of the delay time $\tau$. We also choose $\Omega=\Gamma_{L}=\Gamma_{R}=1$. (b) Qutrit model (see Appendix D): No time delay $\tau=0$. Plot of $\dot{Q}^{L}$ as a function of $T_{L}$, for $T_{R}=1$ and $\alpha=\pi / 3$. Insets: Plot of $\kappa_{C}$ (solid), $\kappa$ (dashed), and $\eta_{C}^{-1}$ (dotted) in the regions where $\dot{Q}^{L}<0$. We choose $\Gamma_{L}^{1}=\Gamma_{R}^{2}=0.1, \Gamma_{L}^{2}=\Gamma_{R}^{1}=2, \Gamma_{L}^{\Delta}=\Gamma_{R}^{\Delta}=0.01, \Omega_{2}=$ $1.1, \Omega_{1}=1.0$.

To operate as a heat pump, the term $\dot{\mathcal{F}}_{E}+T_{R} \dot{\mathcal{F}}_{S}$ generated by the feedback has to be positive since $-\dot{Q}^{L}>0$. As a result by defining the coefficient of performance as

$$
\kappa_{C} \equiv \frac{-\dot{Q}^{L}}{\dot{\mathcal{F}}_{E}+T_{R} \dot{\mathcal{F}}_{S}}=\frac{1}{\eta_{C}}\left(1-\frac{T_{R} \dot{S}_{\mathbf{i}}}{\dot{\mathcal{F}}_{E}+T_{R} \dot{\mathcal{F}}_{S}}\right),
$$

the non-negativity of the entropy production implies that

$$
\kappa_{C} \leqslant \frac{1}{\eta_{C}} .
$$

When $\dot{\mathcal{F}}_{S}=0$, the feedback plays the role of a pure work source and $\kappa_{C}=\tilde{\kappa}$.

In Fig. 3(a), we compare $\kappa_{C}$ with $\tilde{\kappa}$ as a function of the delay time for the qubit model described in Sec. III D. For simplicity we choose the same time delay $\tau$ for all jump types. The qubit operates as a heat pump for positive $\kappa_{C}$ (i.e., negative $\dot{Q}^{L}$ ). The inset shows that for large time delays $\tau \rightarrow \infty$, the effect of the feedback disappears and the energy and entropy contribution of the feedback vanish $\dot{\mathcal{F}}_{E, S} \rightarrow 0$.

A maximum amount of heat is delivered to the left reservoir when $\dot{Q}^{L}=\Omega\left(\gamma_{L} p_{0}-\bar{\gamma}_{L} p_{1}\right)$ is minimized, i.e., 
when $p_{0} \rightarrow 0$ and thus $p_{1} \rightarrow 1$. This can be achieved by choosing a feedback such that $\alpha_{-}^{\nu} \rightarrow \pi / 2, \alpha_{+}^{\nu} \rightarrow 0$ for $v \in$ $\{L, R\}$ [see $(\mathrm{C} 1)]$. In this limit one can even show that the zero delay time limit leads to a vanishing entropy production, $\lim _{\tau \rightarrow 0} \dot{S}_{\mathbf{i}}=0$. Indeed, we can see in Fig. 3(a) that in this reversible limit $\kappa_{C}$ is maximized and reaches its upper bound $1 / \eta_{C}$.

In Fig. 3(b), we use the qutrit model described in Appendix D to show that $\tilde{\kappa}$ can be larger than $\eta_{C}^{-1}$, because this never happens in the qubit model. The insets compare $\kappa_{C}$ with $\tilde{\kappa}$ and $\eta_{C}^{-1}$ as a function of the temperature of the hot reservoir in the regions where the qutrit operates as a heat pump.

\section{B. Stabilization of pure states}

The feedback is able to generate steady state coherences [see Eq. (C2)]. This raises the question whether it is possible to stabilize a pure quantum state. Thus, we are looking for solutions of the equation $0=\mathcal{W}^{C} \rho$ where $\rho$ is a pure state corresponding to $\rho \leftrightarrow \varrho=|\psi\rangle\langle\psi|$ such that $\operatorname{tr}\left[\varrho^{2}\right]=\operatorname{tr}[\varrho]=1$.

Immediately after a quantum jump, the qubit finds itself in an energy eigenstate of the system (which is pure). After the jump, the feedback rotates the qubit into another pure state which in general involves arbitrary superpositions of the energy eigenstates. We denote them as

$$
\left.\left.\mathcal{C}_{+}^{v} \mathcal{J}_{+}^{v}|1\rangle\right\rangle \equiv \rho_{+}^{v}, \quad \mathcal{C}_{-}^{v} \mathcal{J}_{-}^{v}|0\rangle\right\rangle \equiv \rho_{-}^{v} .
$$

For simplicity, we tune the feedback parameters $\left\{\alpha_{ \pm}^{v}\right\}$ so that the state of the system right after the control operation is the same independently of the jump triggering the control operation, i.e., we demand $\rho_{+}^{L}=\rho_{+}^{R}=\rho_{-}^{L}=\rho_{-}^{R}$. By choosing $\alpha_{+}^{v} \equiv \alpha$ and $\alpha_{-}^{v} \equiv \alpha+\frac{\pi}{2}$ we achieve this and the state right after the control operation is (in the ordered eigenbasis $\{|1\rangle,|0\rangle\})$

$$
\varrho_{\text {target }}(\alpha)=\left(\begin{array}{cc}
\cos ^{2} \alpha & i \cos \alpha \sin \alpha \\
-i \cos \alpha \sin \alpha & \sin ^{2} \alpha
\end{array}\right) .
$$

We start by considering the vanishing time delay limit. The system evolution between the jumps is described by the generator $\mathcal{W}_{0}$ and in general destroys coherences as well as the state's purity. A measure of how far the steady state is from $\varrho_{\text {target }}$ is the trace distance. For two arbitrary density matrices $\varrho_{1}$ and $\varrho_{2}$ it is defined by

$$
\mathfrak{D}\left[\varrho_{1}, \varrho_{2}\right] \equiv \frac{1}{2} \operatorname{tr} \sqrt{\left(\varrho_{1}-\varrho_{2}\right)^{2}}=\frac{1}{2} \sum_{i}\left|\lambda_{i}\right|,
$$

where $\lambda_{i}$ are the eigenvalues of $\varrho_{1}-\varrho_{2}$. We have $\mathfrak{D}\left[\varrho_{1}, \varrho_{2}\right] \in$ $[0,1]$. If $\mathfrak{D}\left[\varrho_{1}, \varrho_{2}\right]=0$ the states $\varrho_{1}$ and $\varrho_{2}$ are experimentally indistinguishable.

As we increase the frequency of the jumps (keeping a zero time delay), the absolute magnitude of the terms in $\mathcal{W}_{0}$ will increase but $\mathcal{W}_{0}$ will also have less time to act. This happens for large temperatures when the Bose distributions become very large. The resulting effect on $\mathfrak{D}\left[\varrho_{\text {target }}, \varrho\right]$ is explored numerically in Fig. 4 for $\beta_{L}=\beta_{R} \equiv \beta$. The results for $\beta_{L} \neq \beta_{R}$ are not qualitatively different. As we see, for large temperatures we get closer to $\varrho_{\text {target }}$. In fact, it is even possible to show analytically that

$$
\lim _{\beta_{L}, \beta_{R} \rightarrow 0} \mathfrak{D}\left(\beta_{L}, \beta_{R}, \alpha\right)=0,
$$
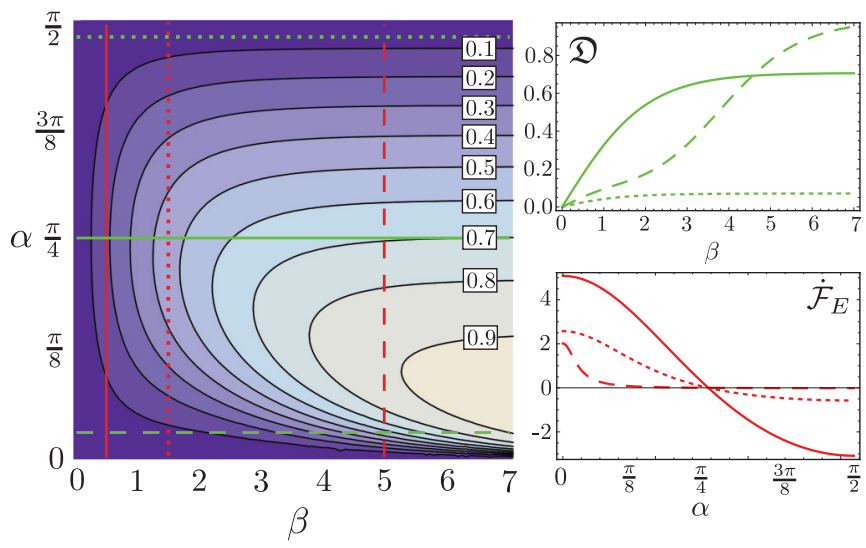

FIG. 4. (Color online) Contour plot of the distance $\mathfrak{D}\left[\varrho_{\text {target }}, \varrho\right]$ for varying $\beta$ and $\alpha$ (left side). The horizontal (green) lines corresponds to a plot of the distance for $\alpha=\pi / 20$ (dashed), $\pi / 4$ (solid), and $3 / 2$ (dotted). The vertical (red) lines corresponds to plots of the energy injection rate due to the feedback for $\beta=0.5$ (solid), 1.5 (dotted), and 5 (dashed). Further values were chosen as $\Gamma_{L}=\Gamma_{R}=1$ and $\Omega=1$.

which implies that the steady state of the system coincides with $\varrho_{\text {target }}$ for any $\alpha$. In this limit, however, also the rate of feedback operations diverges.

We now turn to finite delay times. In Fig. 5 we consider 1 minus the trace distance for the special case $\alpha=\pi / 4$ which corresponds to the state $\varrho_{\text {target }}(\pi / 4)=|\psi\rangle\langle\psi|$ with wave function $|\psi\rangle=\frac{e^{i \varphi}}{\sqrt{2}}(|1\rangle-i|0\rangle)$. As expected, when the time between two subsequent jumps becomes smaller, the influence of the delay becomes stronger. Thus, for finite delay times we observe the appearance of an optimal temperature which maximizes $1-\mathfrak{D}$, i.e., it minimizes the distance between the stationary state and the target state.

At finite temperature, $\mathfrak{D}\left[\varrho_{\text {target }}, \varrho\right]$ strictly vanishes only for $\alpha=0$ and $\alpha=\frac{\pi}{2}$; see Fig. 4 again. This means that one can only fully stabilize the excited state $|1\rangle$ and the ground state $|0\rangle$. To see this theoretically we split the generator as $\mathcal{W}^{C}=\mathcal{W}_{0}+\mathcal{J}^{C}$ where $\mathcal{J}^{C}=\sum_{\nu}\left(\mathcal{C}_{+}^{\nu} \mathcal{J}_{+}^{v}+\mathcal{C}_{-}^{\nu} \mathcal{J}_{-}^{\nu}\right)$ describes the quantum jumps followed by the control operations. The time evolution between the jumps is therefore governed by $\mathcal{W}_{0}$ which, following [28,29], can be expressed by a generalized commutator as $\mathcal{W}_{0} \rho \leftrightarrow-i\left(\tilde{H} \varrho-\varrho \tilde{H}^{\dagger}\right)$, where we introduced

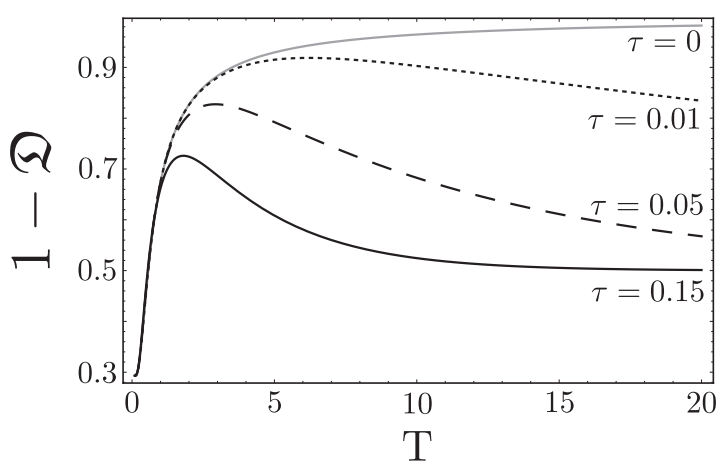

FIG. 5. Plot of $1-\mathfrak{D}\left[\varrho_{\text {target }}, \varrho\right]$ for $\alpha=\pi / 4$ over the common reservoir temperature $T$. All the other parameters are chosen as in Fig. 4. 


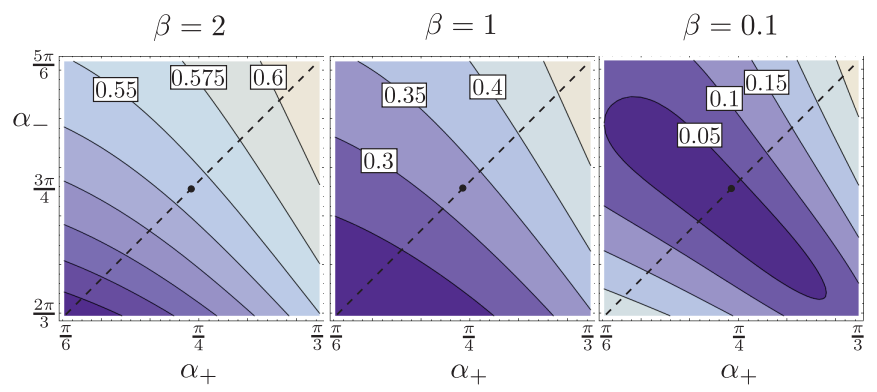

FIG. 6. (Color online) Contour plot of the distance $\mathfrak{D}\left[\varrho, \varrho_{\text {target }}(\pi / 4)\right]$ over three different bath temperatures $\beta_{L}=\beta_{R}=\beta$ where the steady state $\varrho$ is obtained for varying $\alpha_{+}$and $\alpha_{-}$. The dashed diagonal line corresponds to the previous setting $\alpha_{+}=\alpha$ and $\alpha_{-}=\alpha+\pi / 2$ and the dot marks the case $\alpha=\pi / 4$.

an effective non-Hermitian Hamiltonian,

$$
\begin{aligned}
\tilde{H}= & \frac{1}{2}\left[\left(\Omega-i \sum_{v} \bar{\gamma}_{v}\right)|1\rangle\langle 1|\right. \\
& \left.+\left(-\Omega-i \sum_{v} \gamma_{v}\right)|0\rangle\langle 0|\right] .
\end{aligned}
$$

In our example, the Liouville space eigenstates of $\tilde{H}$ and $\tilde{H}^{\dagger}$ are the same and correspond to the Hilbert space eigenstates of the qubit $|0\rangle$ and $|1\rangle$. This means that beside these two states, any other state will be destabilized during the evolution between the jumps.

Until now we tuned the feedback parameters such that the state of the system after any control operation is always the same. We now relax this assumption by considering $\mathfrak{D}\left[\varrho_{\text {target }}, \varrho\right]$ as a function of $\alpha_{+}^{v} \equiv \alpha_{+}$and $\alpha_{-}^{\nu} \equiv \alpha_{-}$where the target state is the pure state $\varrho_{\text {target }}(\pi / 4)$, which corresponds to an equally weighted superposition of the excited state and the ground state. In Fig. 6 we see that-due to the incoherent time evolution between the jumps-it is better to choose slightly different values from $\alpha_{+}=\frac{\pi}{4}$ and $\alpha_{-}=\frac{3 \pi}{4}$ considered before. This discrepancy vanishes for large temperatures.

We finally note that in this section on steady state coherence stabilization, the entropy production (42) always vanished since $\beta_{L}=\beta_{R}$ and $f_{L}=f_{R}$. This shows that the notion of reversibility in the presence of feedback resulting from our treatment clearly does not imply canonical steady states and even allows for pure states. Furthermore, one may even extract energy from the system by stabilizing a certain set of states, as indicated in Fig. 4.

\section{CONCLUSIONS}

We considered open quantum systems in contact with multiple reservoirs and subjected to quantum feedback operations triggered by the detection of transfer processes between the system and its reservoirs. The feedback operations are instantaneous unitary operations in the system Hilbert space, which can be performed immediately after the detection or after a finite time delay.

We showed that the dynamics of such systems can still be analyzed within the framework of stochastic thermodynamics despite the fact that the quantum feedback operation can stabilize coherences in the stationary states which would vanish in the absence of feedback. The quantum feedback operation injects energy and entropy (or information) into the system and thus modifies the energy balance (the first law) as well as the entropy balance (the second law).

In absence of time delay, the effect of the feedback on the thermodynamic description of the system can be understood classically. Each time a monitored reservoir-induced transition occurs in the system, the system ends up in one of its energy eigenstates. The effect of the feedback is to induce a transition between this eigenstate and the other ones with a given transition probability. This results in a change of the energy as well as entropy of the system.

We applied the formalism to study a qubit in contact with two reservoirs and operating as a heat pump. We showed that due to the feedback, the coefficient of performance used to characterize the efficiency of conventional heat pumps is not bounded by the inverse Carnot efficiency anymore. We proposed a new definition of the coefficient of performance that is bounded by our theory. We also analyzed the effect of time delay on the heat pump operation. Finally, we demonstrated that the quantum feedback operation can be used to stabilize coherences in nonequilibrium steady states including pure states.

\section{ACKNOWLEDGMENTS}

Useful discussions with V. Bastidas are acknowledged. Financial support by the DFG (Grants No. SCHA 1646/2-1, No. SFB 910, and No. GRK 1558) and the National Research Fund, Luxembourg (Project No. FNR/A11/02) is gratefully acknowledged.

\section{APPENDIX A: FULL COUNTING STATISTICS}

We derive the currents (7) and (26) using full counting statistics methods [37]. We assign a counting field $\chi_{(i, j)}^{v}$ to every transition from system state $j$ to $i$ due to the reservoir $v$. The corresponding jump operators are denoted $\mathcal{J}_{j \rightarrow i}^{v}$ and $\overline{\mathcal{J}}_{i \rightarrow j}^{v}$. The generator then takes the form

$$
\mathcal{W}(\chi)=\mathcal{W}_{0}+\sum_{\nu} \sum_{i>j}\left(e^{i \chi_{(i, j)}^{v}} \mathcal{J}_{j \rightarrow i}^{v}+e^{-i \chi_{(i, j)}^{v}} \overline{\mathcal{J}}_{i \rightarrow j}^{v}\right) .
$$

If $\chi$ denotes the vector of all counting fields, the formal solution of the density matrix evolved with this generator is

$$
\rho(\chi, t)=e^{\mathcal{W}(\chi) t} \rho(0) .
$$

It turns out that $\operatorname{tr}[\rho(\chi, t)]$ is the moment generating function associated to the net integrated probability currents between the states. Thus, the average net integrated current associated to the transition $j \rightarrow i$ and due to reservoir $v$ is calculated by taking the derivative of the moment generating function with respect to the counting field $\chi_{(i, j)}^{v}$

$$
\langle n\rangle_{(i, j)}^{v}(t)=\left.\frac{\partial}{\partial\left(i \chi_{(i, j)}^{v}\right)} \operatorname{tr}[\rho(\chi, t)]\right|_{\chi=0} .
$$

The time derivative yields the current

$$
I_{(i, j)}^{v}(t)=\frac{\partial}{\partial t}\langle n\rangle_{(i, j)}^{v}(t)
$$


Applying the time derivative to (A2), we obtain

$$
\begin{aligned}
I(t) & =\left.\frac{\partial}{\partial\left(i \chi_{(i, j)}^{\nu}\right)} \operatorname{tr}\left[\mathcal{W}(\chi) e^{\mathcal{W}(\chi) t} \rho(0)\right]\right|_{\chi=0} \\
& =\operatorname{tr}\left[\mathcal{W}^{\prime}(0) e^{\mathcal{W}(0) t} \rho(0)+\mathcal{W}(0)\left(e^{\mathcal{W}(0) t}\right)^{\prime} \rho(0)\right],
\end{aligned}
$$

where $\mathcal{W}^{\prime}(0)$ and $\left(e^{\mathcal{W}(0) t}\right)^{\prime}$ are shorthand notations for the derivative with respect to $\left(i \chi_{(i, j)}^{v}\right)$ evaluated at $\chi=0$. Since the second term vanishes due to the fact that the generator is norm preserving, $\sum_{i} \mathcal{W}_{i j}=0$, and using $\rho(t)=e^{\mathcal{W}(0) t} \rho(0)$ we find that

$$
I_{(i, j)}^{v}(t)=\operatorname{tr}\left[\mathcal{W}^{\prime}(0) \rho(t)\right] .
$$

Using the form of the generator (A1) yields to the desired expression (7).

Reproducing this argument in presence of feedback leads to

$$
I_{(i, j)}^{v}(t)=\operatorname{tr}\left[\left(\mathcal{C}_{j \rightarrow i}^{v} \mathcal{J}_{j \rightarrow i}^{v}-\overline{\mathcal{C}}_{i \rightarrow j}^{v} \overline{\mathcal{J}}_{i \rightarrow j}^{v}\right) \rho(t)\right] .
$$

Evaluating the trace by using the explicit form of the jump operators and (24) gives after some straightforward calculation (26).

\section{APPENDIX B: DERIVATION OF THE FIRST AND SECOND LAW WITH AND WITHOUT FEEDBACK CONTROL}

The time derivative of the average energy of a system described by a rate equation $\dot{p}_{i}(t)=\sum_{j} \mathcal{W}_{i j} p_{j}(t)$ with $\mathcal{W}=$ $\sum_{\nu} \mathcal{W}^{(v)}$ reads

$$
\begin{aligned}
\dot{E}(t) & =\sum_{i} E_{i} \dot{p}_{i}(t) \stackrel{(\star)}{=} \sum_{\nu} \sum_{i, j}\left(E_{i}-E_{j}\right) \mathcal{W}_{i j}^{(\nu)} p_{j}(t) \\
& =\sum_{\nu} \sum_{i>j}\left(E_{i}-E_{j}\right)\left[\mathcal{W}_{i j}^{(\nu)} p_{j}(t)-\mathcal{W}_{j i}^{(v)} p_{i}(t)\right] .
\end{aligned}
$$

For step $(\star)$, we used the fact that the rate equation preserves probability: $\sum_{i} \mathcal{W}_{i j}=0$ for every $j \in\{1, \ldots, M\}$. Using the definition of the heat flow (8), Eq. (B1) immediately gives the first law of thermodynamics without feedback (10). In presence of feedback we have to insert the modified population generator from Eq. (25). This yields after some calculations the expression

$$
\begin{aligned}
\dot{E}(t)= & \sum_{\nu} \sum_{i>j}\left(E_{i}-E_{j}\right)\left[\gamma_{j \rightarrow i} p_{j}(t)-\bar{\gamma}_{i \rightarrow j} p_{i}(t)\right] \\
& +\sum_{\nu} \sum_{i>j}\left\{\left[\sum_{k}\left(\mathcal{C}_{j \rightarrow i}^{v}\right)_{k i} E_{k}-E_{i}\right] \gamma_{j \rightarrow i}^{v} p_{j}(t)\right. \\
& \left.+\left[\sum_{k}\left(\overline{\mathcal{C}}_{j \rightarrow i}^{v}\right)_{k j} E_{k}-E_{j}\right] \bar{\gamma}_{i \rightarrow j}^{v} p_{i}(t)\right\} .
\end{aligned}
$$

The first term equals again the sum over all heat flows and the rest equals the rate of energy injection due to the feedback $\dot{\mathcal{F}}_{E}(t)=\sum_{v} \dot{\mathcal{F}}_{E}^{(v)}(t)$. This can be confirmed by evaluating Eq. (28). Thus, we end up with the first law stated in Eq. (29).

We now turn to the second law of thermodynamics. For this we want to calculate the information flow at steady state. The entropy flow, Eq. (31), can be written after some algebra as

$\dot{S}_{\mathrm{e}}(t)=\sum_{\nu} \sum_{i>j}\left[\left(\mathcal{W}^{C}\right)_{i j}^{(\nu)} p_{j}(t)-\left(\mathcal{W}^{C}\right)_{j i}^{(\nu)} p_{i}(t)\right] \ln \frac{\left(\mathcal{W}^{C}\right)_{j i}^{(\nu)}}{\left(\mathcal{W}^{C}\right)_{i j}^{(\nu)}}$.

In the absence of feedback, using local detailed balance (5) and the definition of the heat flow (8), we get the second law of thermodynamics stated in Eq. (14) with $\dot{S}_{\mathrm{e}}(t)=$ $\sum_{v} \dot{Q}^{(v)}(t) / T_{v}$. In the presence of feedback, we have to use the modified population generator from Eq. (25). After separating the heat flows, we have

$$
\begin{aligned}
\mathcal{F}_{S}^{(v)}(t)-\frac{\dot{Q}^{(v)}(t)}{T_{\nu}}= & -\sum_{m>m^{\prime}}\left(\sum_{i \mid i>m^{\prime}} \gamma_{m^{\prime} \rightarrow i}^{\nu}\left(\mathcal{C}_{m^{\prime} \rightarrow i}^{v}\right)_{m i}+\sum_{j \mid j<m^{\prime}} \bar{\gamma}_{m^{\prime} \rightarrow j}^{v}\left(\overline{\mathcal{C}}_{m^{\prime} \rightarrow j}^{\nu}\right)_{m j}\right) p_{m^{\prime}}(t) \ln \frac{\left(\mathcal{W}^{C}\right)_{m^{\prime} m}^{(v)}}{\left(\mathcal{W}^{C}\right)_{m m^{\prime}}^{(v)}} \\
& +\sum_{m>m^{\prime}}\left(\sum_{i \mid i>m} \gamma_{m \rightarrow i}^{\nu}\left(\mathcal{C}_{m \rightarrow i}^{\nu}\right)_{m^{\prime} i}+\sum_{j \mid j<m} \bar{\gamma}_{m \rightarrow j}^{v}\left(\overline{\mathcal{C}}_{m \rightarrow j}^{\nu}\right)_{m^{\prime} j}\right) p_{m}(t) \ln \frac{\left(\mathcal{W}^{C}\right)_{m^{\prime} m}^{(v)}}{\left(\mathcal{W}^{C}\right)_{m m^{\prime}}^{(v)}}
\end{aligned}
$$

where $\sum_{i \mid i>m}$ denotes a sum running over those $i$ which fulfill $i>m$.

\section{APPENDIX C: STEADY STATE OF THE QUBIT}

For completeness we give the exact steady state of the feedback controlled qubit obtained by solving the equation $0=\mathcal{W}^{C} \rho$ with the generator (38) and (20). The populations read

$$
p_{0}=1-p_{1}=\frac{\bar{\gamma}_{L} \cos ^{2} \alpha_{-}^{L}+\bar{\gamma}_{R} \cos ^{2} \alpha_{-}^{R}}{\gamma_{L} \cos ^{2} \alpha_{+}^{L}+\bar{\gamma}_{L} \cos ^{2} \alpha_{-}^{L}+\gamma_{R} \cos ^{2} \alpha_{+}^{R}+\bar{\gamma}_{R} \cos ^{2} \alpha_{-}^{R}}
$$


and the coherences are given by

$$
\begin{aligned}
\rho_{01}= & \rho_{10}^{*}=\frac{2 i \gamma_{L} \cos \alpha_{+}^{L}\left[\bar{\gamma}_{L} \cos \alpha_{-}^{L} \sin \left(\alpha_{-}^{L}-\alpha_{+}^{L}\right)+\bar{\gamma}_{R} \cos \alpha_{-}^{R} \sin \left(\alpha_{-}^{R}-\alpha_{+}^{L}\right)\right]}{\left(-2 i \Omega+\gamma_{L}+\bar{\gamma}_{L}+\gamma_{R}+\bar{\gamma}_{R}\right)\left(\gamma_{L} \cos ^{2} \alpha_{+}^{L}+\bar{\gamma}_{L} \cos ^{2} \alpha_{-}^{L}+\gamma_{R} \cos ^{2} \alpha_{+}^{R}+\bar{\gamma}_{R}-\bar{\gamma}_{R} \sin ^{2} \alpha_{-}^{R}\right)} \\
& +\frac{2 i \gamma_{R} \cos \alpha_{+}^{R}\left[\bar{\gamma}_{L} \cos \alpha_{-}^{L} \sin \left(\alpha_{-}^{L}-\alpha_{+}^{R}\right)+\bar{\gamma}_{R} \cos \alpha_{-}^{R} \sin \left(\alpha_{-}^{R}-\alpha_{+}^{R}\right)\right]}{\left(-2 i \Omega+\gamma_{L}+\bar{\gamma}_{L}+\gamma_{R}+\bar{\gamma}_{R}\right)\left(\gamma_{L} \cos ^{2} \alpha_{+}^{L}+\bar{\gamma}_{L} \cos ^{2} \alpha_{-}^{L}+\gamma_{R} \cos ^{2} \alpha_{+}^{R}+\bar{\gamma}_{R}-\bar{\gamma}_{R} \sin ^{2} \alpha_{-}^{R}\right)} .
\end{aligned}
$$

\section{APPENDIX D: THE QUTRIT}

The qutrit model is a three-level system with Hamiltonian

$$
H_{S}=\Omega_{2}|2\rangle\left\langle 2\left|+\Omega_{1}\right| 1\right\rangle\langle 1|+0| 0\rangle\langle 0|,
$$

where $\Omega_{2}>\Omega_{1}>0$ defines the levels of the qutrit. As in the qubit model, the interaction in chosen in the RWA

$$
V=\sum_{\nu, \mathbf{q}} \sum_{i<j} T_{\mathbf{q} \nu}\left(b_{\mathbf{q} \nu}^{\dagger}|i\rangle\left\langle j\left|+b_{\mathbf{q} \nu}\right| j\right\rangle\langle i|\right) .
$$

The qutrit is coupled to a left and a right reservoir. The population generator in the ordered basis $\left(p_{0}, p_{1}, p_{2}\right)$ reads

$$
\mathcal{W}_{\text {pop }}=\sum_{v}\left(\begin{array}{ccc}
-\gamma_{v}^{1}-\gamma_{v}^{2} & \bar{\gamma}_{v}^{1} & \bar{\gamma}_{v}^{2} \\
\gamma_{v}^{1} & -\gamma_{v}^{\Delta}-\bar{\gamma}_{v}^{1} & \bar{\gamma}_{v}^{\Delta} \\
\gamma_{v}^{2} & \gamma_{v}^{\Delta} & -\bar{\gamma}_{v}^{2}-\bar{\gamma}_{v}^{\Delta}
\end{array}\right) .
$$

As in the qubit model, the rates are expressed in terms of the Bose distribution $\gamma_{v}^{\omega}=\Gamma_{v}^{\omega} n_{\nu}(\omega), \bar{\gamma}_{v}^{\omega}=\Gamma_{v}^{\omega}\left[1+n_{v}(\omega)\right]$, where $\omega$ denotes an energy difference between system states and we abbreviated $\gamma_{v}^{1} \equiv \gamma_{v}^{\Omega_{1}}, \gamma_{v}^{2} \equiv \gamma_{v}^{\Omega_{2}}, \gamma_{v}^{\Delta} \equiv \gamma_{v}^{\Omega_{2}-\Omega_{1}}$.

We consider the following control scheme. Whenever the transition $|0\rangle \rightarrow|1\rangle$ is detected we apply the control operation $U=\exp [i \alpha(|1\rangle\langle 2|+| 2\rangle\langle 1|)]$. This rotates the level $|1\rangle$ to the superposition $\cos \alpha|1\rangle+i \sin \alpha|2\rangle$. The resulting population generator reads

$$
\mathcal{W}_{\text {pop }}^{C}=\sum_{v}\left(\begin{array}{ccc}
-\gamma_{v}^{1}-\gamma_{v}^{2} & \bar{\gamma}_{v}^{1} & \bar{\gamma}_{v}^{2} \\
\gamma_{v}^{1} \cos ^{2} \alpha & -\gamma_{v}^{\Delta}-\bar{\gamma}_{v}^{1} & \bar{\gamma}_{v}^{\Delta} \\
\gamma_{v}^{1} \sin ^{2} \alpha+\gamma_{v}^{2} & \gamma_{v}^{\Delta} & -\bar{\gamma}_{v}^{2}-\bar{\gamma}_{v}^{\Delta}
\end{array}\right)
$$

[1] H.-P. Breuer and F. Petruccione, The Theory of Open Quantum Systems (Oxford University Press, New York, 2002).

[2] M. Esposito and C. Van den Broeck, Phys. Rev. E 82, 011143 (2010).

[3] M. Esposito and S. Mukamel, Phys. Rev. E 73, 046129 (2006).

[4] G. E. Crooks, J. Stat. Mech. (2008) P10023.

[5] J. Derezinski, W. De Roeck, and C. Maes, J. Stat. Phys. 131, 341 (2008).

[6] J. M. Horowitz, Phys. Rev. E 85, 031110 (2012).

[7] F. W. J. Hekking and J. P. Pekola, Phys. Rev. Lett. 111, 093602 (2013).

[8] G. Schaller, C. Emary, G. Kießlich, and T. Brandes, Phys. Rev. B 84, 085418 (2011).

[9] D. V. Averin, M. Möttönen, and J. P. Pekola, Phys. Rev. B 84, 245448 (2011).

[10] P. Strasberg, G. Schaller, T. Brandes, and M. Esposito, Phys. Rev. Lett. 110, 040601 (2013).

[11] Y. Jun and J. Bechhoefer, Phys. Rev. E 86, 061106 (2012).

[12] D. Abreu and U. Seifert, Europhys. Lett. 94, 10001 (2011).

[13] S. Toyabe, T. Sagawa, M. Ueda, E. Muneyuki, and M. Sano, Nat. Phys. 6, 988 (2010).

[14] M. Esposito and G. Schaller, Europhys. Lett. 99, 30003 (2012).

[15] T. Sagawa and M. Ueda, Phys. Rev. Lett. 100, 080403 (2008).
[16] T. Sagawa and M. Ueda, Phys. Rev. Lett. 104, 090602 (2010).

[17] T. Sagawa and M. Ueda, Phys. Rev. E 85, 021104 (2012).

[18] J. Horowitz and J. M. P. Parrondo, Europhys. Lett. 95, 10005 (2011).

[19] J. M. Horowitz, T. Sagawa, and J. M. R. Parrondo, Phys. Rev. Lett. 111, 010602 (2013).

[20] D. Abreu and U. Seifert, Phys. Rev. Lett. 108, 030601 (2012).

[21] T. Munakata and M. L. Rosinberg, J. Stat. Mech. (2012) P05010.

[22] H. M. Wiseman and G. J. Milburn, Quantum Measurement and Control (Cambridge University Press, Cambridge, England, 2009).

[23] H. M. Wiseman, Phys. Rev. A 49, 2133 (1994).

[24] J. Wang and H. M. Wiseman, Phys. Rev. A 64, 063810 (2001).

[25] C. Sayrin, I. Dotsenko, X. Zhou, B. Peaudecerf, T. Rybarczyk, S. Gleyzes, P. Rouchon, M. Mirrahimi, H. Amini, M. Brune et al., Nature (London) 477, 73 (2011).

[26] D. Ristè, C. C. Bultink, K. W. Lehnert, and L. DiCarlo, Phys. Rev. Lett. 109, 240502 (2012).

[27] P. Campagne-Ibarcq, E. Flurin, N. Roch, D. Darson, P. Morfin, M. Mirrahimi, M. H. Devoret, F. Mallet, and B. Huard, Phys. Rev. X 3, 021008 (2013).

[28] C. Pöltl, C. Emary, and T. Brandes, Phys. Rev. B 84, 085302 (2011). 
[29] G. Kießlich, C. Emary, G. Schaller, and T. Brandes, New J. Phys. 14, 123036 (2012).

[30] D. Segal and A. Nitzan, Phys. Rev. E 73, 026109 (2006).

[31] D. Segal, Phys. Rev. Lett. 101, 260601 (2008).

[32] J. Ren, P. Hänggi, and B. Li, Phys. Rev. Lett. 104, 170601 (2010).

[33] C. Emary, Philos. Trans. R. Soc. A 3711999 (2013).
[34] T. Speck and U. Seifert, J. Stat. Mech. (2007) L09002.

[35] M. Esposito and K. Lindenberg, Phys. Rev. E 77, 051119 (2008).

[36] B. Leggio, A. Napoli, H.-P. Breuer, and A. Messina, Phys. Rev. E 87, 032113 (2013).

[37] M. Esposito, U. Harbola, and S. Mukamel, Rev. Mod. Phys. 81, 1665 (2009). 